\title{
Pathway-based analyses
}

\author{
Jack W. Kent Jr \\ From Genetic Analysis Workshop 19 \\ Vienna, Austria. 24-26 August 2014
}

\begin{abstract}
Background: New technologies for acquisition of genomic data, while offering unprecedented opportunities for genetic discovery, also impose severe burdens of interpretation andpenalties for multiple testing.

Methods: The Pathway-based Analyses Group of the Genetic Analysis Workshop 19 (GAW19) sought reduction of multiple-testing burden through various approaches to aggregation of highdimensional data in pathways informed by prior biological knowledge.

Results: Experimental methods testedincluded the use of "synthetic pathways" (random sets of genes) to estimate power and false-positive error rate of methods applied to simulated data; data reduction via independent components analysis, single-nucleotide polymorphism (SNP)-SNP interaction, and use of gene sets to estimate genetic similarity; and general assessment of the efficacy of prior biological knowledge to reduce the dimensionality of complex genomic data.
\end{abstract}

Conclusions: The work of this group explored several promising approaches to managing high-dimensional data, with the caveat that these methods are necessarily constrained by the quality of external bioinformatic annotation.

\section{Background}

The data provided for Genetic Analysis Workshop 19 (GAW19) offer extensive and complex genomic information (described fully in Blangero et al. [1]). These data included 2 Mexican American cohorts: a set of extended families providing longitudinal data on blood pressure (BP; up to 4 clinic visits per subject) and genomic data including haplotypetagging single nucleotide polymorphisms (SNPs) and whole sequence data for odd-numbered autosomes, as well as gene expression profiles from the first clinic visit; and a separate set of unrelated individuals with $\mathrm{BP}$ and exome sequence data from a single clinic visit. The GAW19 organizers also provided 200 replicates of simulated BP data for both cohorts based on the real genotypes and a polygenic generating model using functional variants in genes chosen based on real associations between $\mathrm{BP}$ and gene expression phenotypes in the Type 2 Diabetes Genetic Exploration by Nextgeneration sequencing in Ethnic Samples (T2D-GENES) study ([1]). This rich genomic resource serves as a sampler of the types of data now becoming available for many study

Correspondence: jkent@txbiomed.org

Department of Genetics, Texas Biomedical Research Institute, PO Box 760549, San Antonio, TX 78245-0549, USA

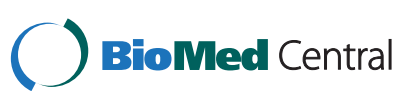

(c) 2016 Kent. Open Access This article is distributed under the terms of the Creative Commons Attribution 4.0 International License (http://creativecommons.org/licenses/by/4.0/), which permits unrestricted use, distribution, and reproduction in any medium, provided you give appropriate credit to the original author(s) and the source, provide a link to the Creative Commons license, and indicate if changes were made. The Creative Commons Public Domain Dedication waiver (http:// creativecommons.org/publicdomain/zero/1.0/) applies to the data made available in this article, unless otherwise stated. cohorts as a result of rapid technological advances (and attendant decreased costs) in acquisition of genomic information.

For GAW19, as in all such studies, this abundance is both a blessing and a curse, a potential source of new insights into the mechanisms of complex disease phenotypes that also introduces an unprecedented burden of correction for multiple tests and interpretation of results. A class of emerging techniques for managing this abundance involves narrowing the search space by grouping the units of analysis (gene expression probes, sequence variants, etc) into biologically relevant pathways (see, eg, Wang et al [2] for a review of software for pathway analysis to follow genome-wide association studies [GWAS]). Pathway analysis can proceed along at least 2 general lines [3]:

1. Preselection of biological pathways believed to be relevant to the disease or phenotype of interest. The primary goals of this approach are to limit the number of genomic features, and therefore the multiple-testing burden, to those annotated to genes in the candidate pathways, and/or to confirm the prior biological hypotheses. An additional aim is to manage genetic heterogeneity: for example, different 
lineages may segregate variation in different genes in the same pathway that nonetheless yield similar biological effects. Without prior biological knowledge, this heterogeneity might be interpreted as noise rather than as concordant signal [4].

2. Gene enrichment tests of genomic features prioritized by evidence of association with the phenotype of interest to identify biological pathways a posteriori. The primary goal of this approach is to interpret the biological significance of findings from agnostic tests of association.

Both of these lines of investigation are constrained by existing biological knowledge (and by the curation strategies and quality of available bioinformatic databases).

Because pathway analysis is relatively new, methods for assigning genomic features to pathways and for testing the significance of these assignments in relation to phenotypes are still very much under development. This developmental fluidity was the context for this discussion group at GAW19.

\section{Methods}

This report is based on the work of 7 research teams who presented their work in our group discussion at GAW19. In the following discussion, these teams are referenced by the name of the presenting author, as shown in Table 1.

Tables 2 and 3 summarizes the teams' approaches to the group topic, including the focal phenotypes chosen from the GAW19 data, the focal genomic feature (gene expression or genetic variants), and the analytical tools employed. These choices, which were intimately associated with each team's primary aims, are compared and discussed in detail under Results.

\section{Results and discussion Motivation}

An overriding motivation for employing pathway analysis was to reduce the multiple-testing burden by basing inferences on the combined effects of probes or variants. Most teams used external bioinformatic data to inform pathway construction (see Table 3). However, the use of pathways to clarify biological function or interpret association results, while highly relevant to future applications of these methods, was a secondary concern given the methoddevelopment focus of these studies. Of all the studies, Ziyatdinov came closest to this interpretive approach, using relationships in the data to recover the biological processes implicit in the simulation generating model.

\section{Data selection; pathway assembly}

All teams used the BP data (real or simulated) provided for GAW19, including systolic (SBP) and diastolic BP (DBP) and hypertension (HT) status. Several teams also calculated the derived phenotype mean arterial pressure (MAP) from these data as $(2 / 3 \mathrm{DBP}+1 / 3 \mathrm{SBP})$. All teams using the simulated data chose not to be blind to the generating model (they "used the answers"), which allowed them to estimate power to detect "true" functional variants and associated gene expression phenotypes. Most teams also used the provided null trait Q1 to estimate type 1 error rate. A limitation of this use of the simulated data (as noted by a reviewer of an early draft of this report) is that evaluation of method performance is necessarily sensitive to the assumptions of the generating model.

Three teams (Brunel, Quillen, Ziyatdinov) focused on the gene expression probes, while the remaining teams considered single nucleotide variants (SNVs) drawn from the GWAS genotypes, exome sequence data, or both. Combined analysis of gene expression and association has been considered in previous GAWs (eg, Charlesworth et al. [5]), but only Brunel attempted this combination in a 2-step approach (data reduction of BP-related gene expression phenotypes followed by GWAS).

As noted, most teams made use of the simulated phenotypes as a basis for estimating power and type 1 error. Kos,

Table 1 Research teams participating in the pathway-based analyses group

\begin{tabular}{lll}
\hline $\begin{array}{l}\text { Presenting } \\
\text { author }\end{array}$ & Abstract Title/Coauthors & Reference \\
\hline Brunel H & $\begin{array}{l}\text { Meta-expression: A methodology for } \\
\text { the joint analysis of gene expression } \\
\text { in biological pathways. }\end{array}$ & Unpublished \\
& $\begin{array}{l}\text { Massanet R, Fernández-Albert F, } \\
\text { Ziyatdinov A, Soria JM, Perera A }\end{array}$ & \\
& &
\end{tabular}

Kos MZ Comparison of GWAS and exome

Unpublished sequence data sets from GAW19. Blackburn AN, Almasy L

Lo A Network-guided interaction mining for blood pressure phenotype of unrelated individuals in GAW19.

Agne M, Auerbach J, Fan R, Lo S-H, Wang $P$, Zhang $T$

Quillen EE A variance component method for integrated pathway analysis of gene expression data. Blangero J, Almasy L

Tayo BO Association of polymorphisms in the aldosterone-regulated sodium reabsorption pathway with blood pressure among Hispanics. Tong L, Cooper RS

Valcarcel A A hierarchical approach to SNP-set analysis: An evaluation of power and type 1 error of gene-based tests of association after pathway-based analysis. Grinde K, Cook K, Green A, Tintle N

Ziyatdinov A Prioritization strategies in enrichment analysis of gene expression data in GAW19 family-based study.

Fernández-Albert F, Vazquez-Santiago M, Soria JM, Perera-Lluna A

Unpublished

unpublished \\ .
}

(a)


Table 2 Summary of research approaches: Data used

\begin{tabular}{|c|c|c|c|c|}
\hline Contribution & Genomic data & Cohort & Phenotype category & Phenotype(s) \\
\hline Brunel & Gene expression/GWAS & Families & Real (gene expression) & Derived $^{a}$ \\
\hline Kos & GWAS and exomic variants & Families & Simulated $^{\mathrm{b}}$ & DBP (first visit) \\
\hline Lo & GWAS and exomic variants & Unrelated & Real & DBP (first visit) \\
\hline Quillen & Gene expression & Families & Simulated $^{\mathrm{b}}$ & DBP (first visit) \\
\hline Tayo & GWAS and exomic variants & Unrelated & Simulated ${ }^{\mathrm{b}}$ and real & $\mathrm{DBP}, \mathrm{SBP}, \mathrm{HT}, \mathrm{MAP}$ \\
\hline Valcarcel & GWAS and exomic variants & Families & Simulated ${ }^{b}$ & Means across visits of DBP, SBP, HT, MAP \\
\hline Ziyatdinov & Gene expression & Families & Real & SBP \\
\hline
\end{tabular}

DBP diastolic blood pressure; GWAS, genome-wide association studies; HT hypertension; MAP mean arterial pressure; SBP, systolic blood pressure

andependent components (see Table 3 and text)

bAuthors were aware of simulation model "answers"

Quillen, and Valcarcel constructed synthetic pathways (described more fully below) of gene expression probes (Quillen) or SNVs (Kos, Valcarcel) representing predetermined numbers of randomly chosen genes; these synthetic pathways were then characterized by the number of genes that contributed to the simulated phenotypes as a basis for measuring the effect of functional "dosage" on analytical power. One team (Tayo) focused on a single biological pathway with known relevance to hypertension (aldosterone-regulated sodium reabsorption), using both real and simulated BP phenotypes.

Kos, Quillen, and Valcarcel independently conceived of using sets of probes or variants annotated to randomly selected genes to define synthetic pathways (SPs) to test both the power and type 1 error of their proposed methods. SPs that represented genes used in the simulation generating model were, in Quillen's terminology, "positive controls" to test the sensitivity of tests. SPs lacking such representation provided data on false positive rate, either in place of or supplementary to tests of the null trait Q1. All 3 teams used various metrics-for example, number of genes represented in each SP or number of generating-model genes represented-to further characterize the performance of their analytical approaches.

In addition to the dimensional reduction achieved by grouping probes or variants in pathways, some teams also filtered variants based on predicted function (eg, Kos).

Lo developed biological pathways nominally based on a hypothesis that hypertension could be related to undetected type 2 diabetes (this is known to have elevated prevalence in the Mexican American cohorts from which the GAW19 data were derived, but diabetes status was not reported as part of the provided data set). Pathway construction consisted of choosing a set of diabetes-related genes from the literature and then expanding these "seed" genes with publicly available gene interaction data. In the context of this workshop, this approach simply provided a biologically plausible way to construct pathways reflecting the number of genes and patterns of gene-gene interaction that might be encountered in a "real" study; this was tangential to the primary goal of characterizing performance of these investigators' bespoke method for analyzing SNP-SNP interaction effects [6] (see below).

As noted, most pathways were user-defined, either based on numbers of genes (to test effects on power and

Table 3 Summary of research approaches: Analytical strategies

\begin{tabular}{|c|c|c|c|c|}
\hline Contribution & Pathway assembly & Probe/variant filters & $\begin{array}{l}\text { Source of annotations/ biological } \\
\text { significance }\end{array}$ & Analytical tools \\
\hline Brunel & $\begin{array}{l}\text { Annotated to BP genes and } \\
\text { interactors }\end{array}$ & $\begin{array}{l}\text { Ontology, protein-protein } \\
\text { interaction }\end{array}$ & Gene Ontology, other (unspecified) & $\begin{array}{l}\text { Independent components } \\
\text { analysis, GWAS in GenABEL }\end{array}$ \\
\hline Kos & Synthetic & $\begin{array}{l}\text { Nonsynonymous or stop } \\
\text { codon gain/loss }\end{array}$ & $\begin{array}{l}\text { KEGG, Biocarta, Pathway Interaction } \\
\text { Database, Reactome }\end{array}$ & $\begin{array}{l}\text { SOLAR measured genotype } \\
\text { association }\end{array}$ \\
\hline Lo & $\begin{array}{l}\text { Annotated to type } 2 \text { diabetes } \\
\text { genes and interactors }\end{array}$ & Not applicable & OMIM, GeneMania & $\begin{array}{l}\text { Bespoke method to detect SNP- } \\
\text { SNP interaction }\end{array}$ \\
\hline Quillen & Synthetic & Not applicable & Ariadne Pathway Studio 8.0 & $\begin{array}{l}\text { Empirical similarity matrices, } \\
\text { variance decomposition }\end{array}$ \\
\hline Tayo & $\begin{array}{l}\text { Annotated to genes in specific } \\
\text { pathway }\end{array}$ & $\begin{array}{l}\text { BP-related pathway of } \\
\text { interest }\end{array}$ & KEGG & PLINK association \\
\hline Valcarcel & Synthetic & Not reported & ANNOVAR & SKAT, burden tests \\
\hline Ziyatdinov & Associated with BP & Heritability & Gene Ontology & Linear mixed models \\
\hline
\end{tabular}


specificity) or on prior biological information, rather than on empirical networks (eg, by weighted correlation networks) [7]. This was consistent with the emphasis on methodological development, especially on data reduction and control of the burden of multiple testing. The known causal structure of the simulated data might have supported a test of the efficacy of an empirical networks approach to recover the generating model from patterns of SNV association, but no team attempted this.

\section{Analytical tools}

The focus for methodological innovation in these studies was typically on the construction or definition of pathways. In general, established tools were used for relating pathway-defined probes or variants to the traits of interest, accounting for family data where appropriate: for example, variance components-based analyses implemented in SOLAR (Sequential Oligogenic Linkage Analysis Routines) [8], by Kos and Quillen; adaptation of SKAT (sequence kernel association test) by Valcarcel; burden tests [9]; GenABEL [10] by Brunel; and PLINK for unrelated data [11].

An exception to this pattern was the use by Lo of an analytical tool to detect gene interactions, previously developed by members of this team [6]. In brief, this method involves comparing the joint effect of 2 SNPs on a trait to the marginal effect of each; a ratio of joint to marginal effects significantly greater than 1 (as assessed by permutation) is taken as evidence of SNP-SNP interaction effects on the phenotype [6]. Using pairwise tests of SNPs annotated to genes in a pathway of interest (described above), these authors reported evidence of a joint effect of variants in GCK and PAX4 on hypertension (real data from the unrelated cohort). As noted by the anonymous reviewers of these authors' report, the nature of this joint effect-whether due to in-sample LD, interaction between the gene products, or some other effect-needs additional investigation.

\section{Gene expression}

We now consider selected approaches in more detail. Taking replicate simulations of DBP as the trait of interest, Quillen analyzed the random effect of similarity in gene expression among related individuals, with the goal of identifying pathways for which coexpression accounted for a significant proportion of variance relative to modeling the random effect of kinship alone. This analysis compared the effect of 16 methods for computing similarity matrices from the expression data; of these, simple correlation and extended Jaccard distance [12] outperformed the others and greatly exceeded the performance of single-probe association as a consequence of reduced multiple testing. As expected, performance was dependent on the number of genes in the SPs that contributed by design to the simulated phenotypes [13].

Brunel developed pathways based on probes in the GAW19 data that were annotated to genes related to BP in Gene Ontology, or to genes related to these by published evidence of protein-protein interaction. They then used independent components analysis (ICA) to derive "meta-expression" phenotypes (ICA factors). Unfortunately, the use of phenotypes derived from the real gene expression data, rather than simulated data, prevented estimation of power and type 1 error rate, so it was not possible to determine if any increase in signal from data reduction could overcome the multipletesting burden inherent in GWAS.

\section{Single nucleotide variants}

Valcarcel proposed 2-step association tests of a simulated BP phenotype that consisted of variant aggregation (via either SKAT or burden test) for all variants in a pathway, followed by gene-centric aggregation tests within pathways that passed a chosen significance threshold. Their pathways were synthetic (randomly chosen sets of specified numbers of genes with retention of pathways that contained at least 1 gene that was causal in the simulation). The 2-step approach was more powerful, with reasonable control of type 1 error, than a 1-step gene-centric approach because of the reduction in multiple testing by prescreening of pathways.

\section{Conclusions}

A primary focus in the Pathway-based Analyses Group was on managing the very large dimensionality of -omics data to increase analytical power. As noted in the Introduction, pathway analysis can also be employed for biological discovery, although this use was not focal for participants in this discussion group. Methodology for this sort of discovery is also in its infancy and would also be a worthy topic for future investigation, although provision of optimal simulation data for this purpose (eg, simulated gene expression data) would be a major challenge.

Because many pathway-based approaches, including the pathway construction carried out by participants in this group, are heavily dependent on bioinformatic databases, our group discussions frequently returned to concerns about the current state of these resources, no matter how well curated - including inaccuracies, difficulty of interpretation, and (inevitably) publication bias. This suggests a possible contradiction at the heart of pathway analysis (which, regrettably, we did not discuss): many of our studies used pathway aggregation to reduce the multipletesting burden that hampers gene and variant discovery, yet pathway construction based on prior knowledge runs the risk of constraining the scope of discovery. A possible 
way out of this dilemma, as noted above, is to search for empirical networks that may reveal unexpected gene interactions and thus novel biological insight.

\section{Competing interests}

The author declares that he has no competing interests.

\section{Authors' contributions}

JWK wrote the manuscript, acknowledging the extensive discussion within our GAW19 group and the individual contributions published separately and cited in the References.

\section{Acknowledgements}

I am grateful to all of the Pathway-based Analyses Group members for their work and participation, and to Dr. Duncan Thomas and 2 anonymous reviewers for their careful reviews of the first draft of this paper. The Genetic Analysis Workshops are supported by National Institutes of Health $(\mathrm{NIH})$ grant GM031575. My participation in GAW19 was supported by NIH grant DK084289.

\section{Declarations}

This article has been published as part of BMC Genetics Volume 17 Supplement 2, 2016: Genetic Analysis Workshop 19: Sequence, Blood Pressure and Expression Data. Summary articles. The full contents of the supplement are available online at www.biomedcentral.com/bmcgenet/ supplements/17/S2. Publication of the proceedings of Genetic Analysis Workshop 19 was supported by National Institutes of Health grant R01 GM031575.

Published: 3 February 2016

\section{References}

1. Blangero J, Teslovich TM, Sim X, Almeida MA, Jun G, Dyer TD, et al. Omics-squared: human genomic, transcriptomic and phenotypic data for Genetic Analysis Workshop 19. BMC Proc. 2015;9 Suppl 8:S2

2. Wang K, Li M, Hakonarson H. Analysing biological pathways in genome-wide association studies. Nat Rev Genet. 2010:11:843-54.

3. Cantor RM, Lange K, Sinsheimer JS. Prioritizing GWAS results: a review of statistical methods and recommendations for their application. Am J Hum Genet. 2010;86:6-22

4. Basson J, Simino J, Rao DC. Between candidate genes and whole genomes: time for alternative approaches in blood pressure genetics. Curr Hypertens Rep. 2012;14:46-61.

5. Charlesworth JC, Peralta JM, Drigalenko E, Göring HHH, Almasy L, Dyer TD, et al. Toward the identification of causal genes in complex diseases: a gene-centric joint test of significance combining genomic and transcriptomic data. BMC Proc 2009;3 Suppl 7:592.

6. Lo S, Chernoff H, Cong L, Ding $Y$, Zheng T. Discovering interactions among BRCA1 and other candidate genes associated with sporadic breast cancer. Proc Natl Acad Sci U S A. 2008;105(34):12387-92.

7. Langfelder P, Horvath S. WGCNA: an R package for weighted correlation network analysis. BMC Bioinformatics. 2008:9:559.

8. Almasy $\mathrm{L}$, Blangero J. Multipoint quantitative trait linkage analysis in general pedigrees. Am J Hum Genet. 1998;62:1198-211.

9. Valcarcel A, Grinde K, Cook K, Green A, Tintle N. A multi-step approach to SNP-set analysis: an evaluation of power and type I error of gene-based tests of association after pathway-based association tests. BMC Proc. 2015;9 Suppl 8:S49

10. Aulchenko YS, de Koning DJ, Haley C. Genome-wide rapid association using mixed model and regression: a fast and simple method for genome-wide pedigree-based quantitative trait loci association analysis. Genetics. 2007;77(1):577-85

11. Tayo BO, Tong L, Cooper RS. Association of polymorphisms in the aldosterone-regulated sodium reabsorption pathway with blood pressure among Hispanics. BMC Proc. 2015;9 Suppl 8:S48.

12. Strehl A, Ghosh J. Value-based customer grouping from large retail data-sets. In: Proceedings of the SPIE Conference on Data Mining and Knowledge Discovery, Orlando, vol. 4057. 2000. p. 33-42.

13. Quillen EE, Blangero J, Almasy L. A variance component method for integrated pathway analysis of gene expression data. BMC Proc. 2015;9 Suppl 8:S47

\section{Submit your next manuscript to BioMed Central and take full advantage of:}

- Convenient online submission

- Thorough peer review

- No space constraints or color figure charges

- Immediate publication on acceptance

- Inclusion in PubMed, CAS, Scopus and Google Scholar

- Research which is freely available for redistribution

Submit your manuscript at www biomedcentral.com/submit
C Biomed Central 